\title{
Tendencias, desafíos y desarrollos de la educación a distancia y virtual en la universidad venezolana
}

\author{
IVORY MOGOLLÓN DE LUGO
}

Doctora en Educación. Tecnología instruccional y educación a distancia por NOVA Southearstern University, Estados Unidos de Norteamérica. Magister en Psicología, Universidad Simón Bolívar, Venezuela. Especialista en Dinámica de Grupos, Universidad

Central de Venezuela, México. Educadora y orientadora, Universidad Central de Venezuela, psicopedagoga, Escuela Superior de Psicopedagogía. Certificado en Liderazgo en Educación a Distancia de la NOVA Southearstern University, NSU/CREAD. Diplomado en Evaluación de la Calidad Educativa, CREFAL, Diplomados en Evaluación de la calidad de programas y cursos a distancia, CALED, UTPL, Ecuador. Premio Andrés Bello 2005, UDUAL. Líneas de investigación: educación a distancia, tecnologías, comunicación, evaluación, formación docente, liderazgo.

Resumen

Las tendencias, los desafíos y desarrollos del presente milenio han traído un interesante espacio de reflexión y análisis sobre los retos, y las oportunidades que plantea la cuarta Revolución Industrial para la educación y para la formación del talento humano hacia la innovación. Las universidades están en la obligación de actuar para responder a las exigencias del mundo actual, avanzar y superar la resistencia al cambio y lograr su transformación, respondiendo a los señalamientos del Informe Horizon y lineamientos de la UNESCO en la Declaración en Incheon para la Educación 2030. En este artículo se intenta responder cómo avanza la universidad venezolana con las exigencias del milenio.

Palabras clave

Tecnologías; Transformación tecnológica; Educación; Cambio. 


\title{
Trends, challenges and developments of the distance and virtual education in the venezuelan university
}

\begin{abstract}
The trends, challenges and developments of the distance and virtual education in this millennium have created an interesting space for reflection and analysis about the objectives and opportunities that the Fourth Industrial Revolution considers for education and human talent training on the way of innovation. Universities must act to respond to the demands of today's world, overcome the resistance to change, and achieve its transformation, based on the indications of the Horizon Report and UNESCO's guidelines in the Education 2030-Incheon Declaration. This article attempts to answer how the Universidad Central de Venezuela advances with the demands of the millennium.
\end{abstract}

Keywords

Technologies; Technological Transformation; Education; Change.

\section{Tendências, desafios e desenvolvimentos da educação a distância e virtual na universidade venezuelana}

\section{Sumário}

As tendências, os desafios e os desenvolvimentos do presente milênio trouxeram um interessante espaço de reflexão e análise sobre os reptos e as oportunidades que apresenta a Quarta Revolução Industrial para a educação e a formação do talento humano na inovação. As universidades têm a obrigação de agir para responder às exigências do mundo atual, avançar e superar a resistência à mudança, e alcançar sua transformação respondendo aos sinais do Relatório Horizon e às diretrizes da UNESCO na Declaração em Incheon para a Educação 2030. Este artigo tenta responder como avança a universidade venezuelana perante as demandas do milênio.

\section{Palavras chave}

Tecnologias; Transformação Tecnológica; Educação; Mudança. 


\section{Introducción}

Las tecnologías de la información y comunicación han configurado a la sociedad desde finales del siglo pasado, lo que implica que se han instaurado en este siglo XXI con la cuarta Revolución Industrial. La universidad, en este sentido, ha incorporado paulatinamente las mismas a su quehacer y en la actualidad explora hasta las tecnologías emergentes para dar respuesta a este inminente desafío.

Como se ha observado en el tiempo, las tecnologías transformaron las formas de entender la comunicación, lo que ha llevado a la desaparición del espacio-tiempo en el intercambio que se establece en los procesos de comunicación por la inmediatez con que ellas permiten que se realicen. Esta influencia de las tecnologías ha transformado las relaciones laborales, interpersonales, la cultura, la economía, la educación y todos los diferentes ámbitos de acción del ser humano.

No solo se habla de nativos digitales, de millennials, sino que las tecnologías se han adoptado como formas de comunicación entre las distintas generaciones. De acuerdo a esto, se puede hablar de una maduración digital. Si esto es así, la universidad está obligada a avanzar y superar la resistencia al cambio y a lograr su transformación tecnológica, ya que como es conocido por experiencias mundiales, la universidad ha tendido a resistirse a cualquier tipo de innovación tecnológica. Ahora bien, el cambio es inminente, hay un reconocimiento de la existencia de un movimiento innovador al cual la universidad no puede resistirse.

Con base en esto, se han generado cambios estructurales para continuar en esta corriente que socialmente impulsa la incorporación y transformación tecnológica. En las universidades, ya no es posible la no utilización de la tecnología. En estos espacios académicos se han diseñado campus virtuales, aplicaciones web específicas para mejorar los procesos administrativos, docentes y estudiantiles, por mencionar algunos. De igual manera, las tecnologías emergentes tales como inteligencia artificial, big data, asistentes virtuales, realidad aumentada, analíticas de aprendizajes, entre otras, están a la orden y disposición de la universidad.

Sin embargo, existen rezagos en algunas universidades venezolanas que todavía no incorporan totalmente la oferta de estudios por medio de estas tec- 


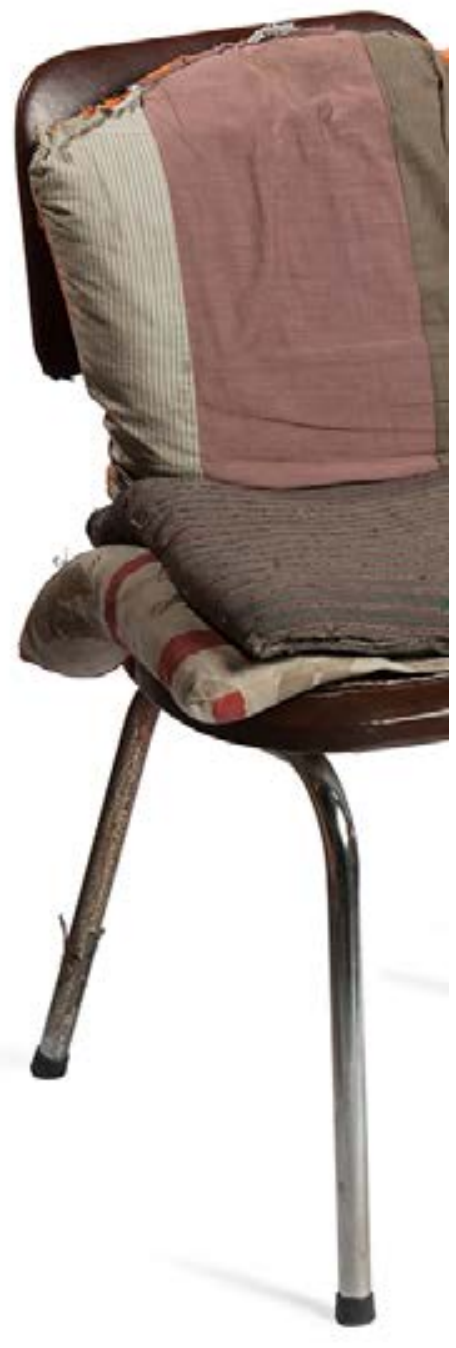

0

Universidades $\mid$ núm. 83, enero-marzo 2020 | UDUAL | DOI.https://doi.org/10.36888/udual.universidades.2020.83.73 Ivory Mogollón de Lugo Tendencias, desafíos y desarrollos de la educación a distancia y virtual en la universidad venezolana nologías y quedan en lo fundamental. La universidad venezolana ha hecho esfuerzos considerables por mantenerse a niveles de transformación tecnológica moderada, ha incorporado con timidez la innovación, y ha asumido retos controlados. Además, estas incorporaciones implican inversiones importantes en laboratorios, en investigación, en formación, en infraestructura, esto también lleva consigo un cambio de cultura y del quehacer universitario.

Ahora bien, la mayoría de las universidades venezolanas son mayoritariamente presenciales, su incursión en las tecnologías tuvo avances importantes a finales del siglo xx y se potenciaron en la primera década del presente siglo con la creación de sistemas de educación a distancia en las universidades más reconocidas del país. Esto hizo que las universidades realizaran cambios en su estructura organizacional y crearan dependencias que respondieran a la misión y visión de esos sistemas. Sin embargo, ese repunte ha disminuido en los últimos años, aunque la universidad hace esfuerzos por mantenerse en las tendencias tecnológicas mediante investigaciones, formación docente, actualización de sus campus virtuales, transformación tecnológica, entre otros, para responder a los señalamientos del Informe Horizon, el cual contiene lineamientos de la UNESCO; a este documento también se le conoce como Declaración en Incheon.

\section{Tendencias y cambio institucional}

El análisis de las tendencias tecnológicas y su aplicación a la universidad en sus funciones de docencia, investigación, extensión y gestión en este mundo de constantes cambios y que hacen necesaria la adaptación para la supervivencia, la motivación surge como un estímulo fundamental para este cambio institucional.

La integración de las tecnologías a la totalidad de las universidades venezolanas se realiza con la intención de favorecer la inclusión en el sistema de educación superior y busca garantizar la calidad en términos de eficacia, eficiencia y pertenencia (Mogollón, 2016) como lo ha promulgado la UNESCO en esta última década. Por otra parte, el Informe Horizon del 2019 presenta seis tendencias claves, seis desafíos significativos y seis desarrollos de tecnologías con impacto en la educación superior en los próximos cinco años. Las seis tendencias claves que 
aceleran la adopción de la tecnología en la educación superior son en primer lugar, repensar cómo funcionan las Instituciones de Educación Superior (IES), y en segundo lugar, ofrecer títulos por módulos y desagregados. Estas tendencias corresponden a cómo satisfacer las necesidades académicas y sociales de los estudiantes, cambios en el rol del docente, programas académicos, incremento de programas en línea, estudios interdisciplinarios y enfoque multidisciplinarios, asociados con titulaciones más allá de las tradicionales con una estructura modular que le ofrece al estudiante la oportunidad de obtener títulos y credenciales particulares combinando las rutas tradicionales con las nuevas.

En tercer lugar, se espera avanzar en la cultura de la innovación y, en el cuarto lugar, mostrar un interés creciente en la medición del aprendizaje. Esto vendría dado por la creación de una cultura de innovación con el desarrollo de habilidades que no solo estén relacionadas a los campos disciplinarios convencionales sino focalizados en la formación para el trabajo y el mundo laboral. Y en lo que se refiere a la medición del aprendizaje, los ambientes digitales brindan la oportunidad para valorar, medir y documentar los aprendizajes.

En quinto lugar, se promueve al rediseño de los espacios de aprendizaje y en sexto lugar, se incorporan los diseños de aprendizajes híbridos, mixtos o blended. Esto requiere de una inversión y planeamiento estratégico que ha sido iniciada en las IES. Estas tendencias se esperan alcanzar a corto plazo debido a que el creciente aprendizaje en línea y el desarrollo de recursos como la realidad aumentada ya están presentes. Por lo tanto, la cualificación de los docentes es una necesidad inminente. También se ha hecho evidente el desarrollo de entornos virtuales de aprendizaje, ricos en medios y herramientas que sirven de soporte al proceso educativo virtual en sus diferentes modalidades.

Los seis desafíos que impiden la adopción de las tecnologías en la educación superior se han dividido en solucionables, difíciles y muy difíciles. Entre los solucionables se pueden mencionar: mejorar la fluidez digital, responder a la demanda de experiencias de aprendizaje digital y de experticia en diseños de instrucción digitales; esto se refiere a la alfabetización digital y al uso de herramientas de aprendizaje con tecnologías sofisticadas que crean la necesidad de que estudiantes y docentes desarrollen nuevas

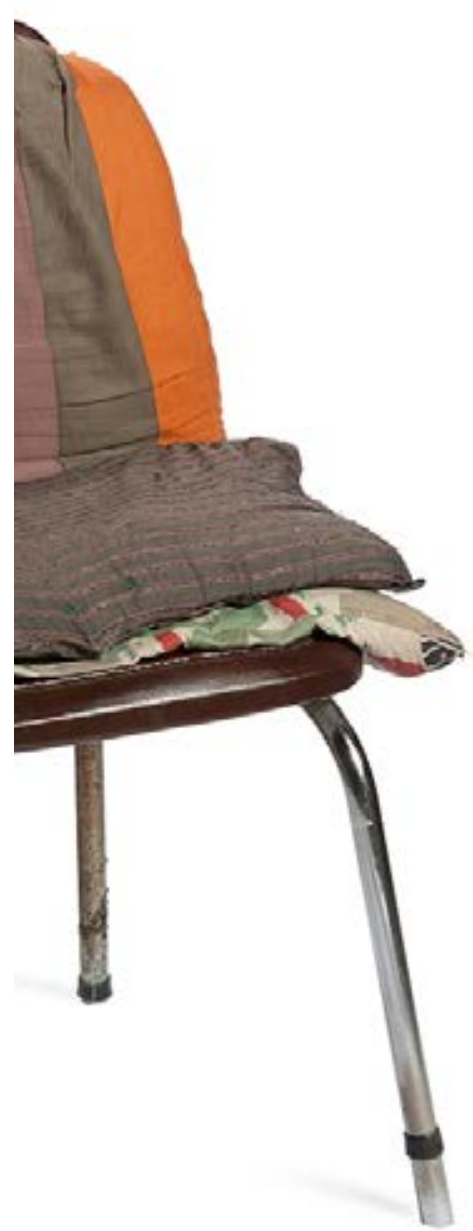


habilidades y trabajen con ellas de manera productiva, con foco en el pensamiento crítico y la solución de problemas.

Entre los desafíos difíciles, están el cambio constante del rol del docente con los avances tecnológicos y la brecha para alcanzar los logros educativos. El docente debe permanecer actualizado y formado en el diseño de estrategias aplicadas al aprendizaje digital. Además, para superar la brecha se requiere de alta conectividad entre todos los actores del proceso educativo, así como de disponibilidad de hardware, acceso a internet libre para acceder a bibliotecas y otros espacios investigativos.

Desafíos muy difíciles son aquellos que resaltan cómo avanzar en la equidad digital y repensar las prácticas de la enseñanza. El primero está en la posibilidad de disponer de un acceso seguro y garante de participación en la Web. El segundo tiene que ver con repensar el rol del docente, cambiar del diseño de cursos, usar las herramientas digitales, los enfoques de aprendizaje activo, modos de evaluar, apoyo y acceso sostenible a los recursos y la creación de experiencias innovadoras.

De los seis desarrollos de tecnologías educativas algunos incluyen el aprendizaje móvil y las tecnologías analíticas. El aprendizaje móvil se focaliza en la conectividad y en experiencias de aprendizaje con contenidos, sincronización y acceso a la información en todo tiempo y lugar. La realidad aumentada ha permitido que el aprendizaje móvil sea más activo y colaborativo. Las tecnologías analíticas trasforman de manera sustancial los procesos académicos, administrativos y financieros.

Otros desarrollos trabajan la realidad mezclada, la inteligencia artificial. Esta denominación de realidad mezclada viene dada por la coexistencia de objetos físicos y virtuales en un espacio híbrido para la comprensión de los fenómenos reales observables y no visibles como el uso de hologramas, la creación de laboratorios, las simulaciones, entre otros. La inteligencia artificial como el uso de reconocimiento facial, personalizar experiencias, reducir cargas de trabajo, producir aplicaciones científicas, educativas, también se encuentran entre dichos desarrollos.

Para los desarrollos a mediano plazo se identifican los blockchains y los asistentes virtuales. Con la primera herramienta se crea una estructura segura para la custodia de información académica y personal. Esto está en pleno desarrollo. En educación están asociados a las calificaciones, expedientes académicos, constancias de títulos, el registro de certificaciones académicas y laborales a la disposición en cualquier lugar y momento.

Los asistentes virtuales apoyados en la inteligencia artificial como reconocimiento de voz, el proceso natural del lenguaje que ya se encuentra en los dispositivos móviles como Siri, Alexa o Bixby favorecen y agilizan los procesos académicos, administrativos y financieros de las IES. Como se puede evidenciar, el Infome Horizon recorre un camino que ya transitan las universidades del mundo y otros aspectos que están en desarrollo y son desafíos para la educación superior.

Las universidades venezolanas en la actualidad realizan enormes esfuerzos por alcanzar estas actualizaciones, se observan incursiones puntuales desde las IES mediante investigaciones que aportan productos que favorecen los procesos académicos, alianzas con consorcios y organismos que apoyan en alcanzar los avances científicos, humanísticos y tecnológi- 
cos. Sin embargo, es innegable que las inversiones son escasas, los presupuestos han disminuido considerablemente, la diáspora del talento humano calificado ha impactado en la productividad y alto desempeño de las universidades venezolanas.

\section{¿Cómo avanza la universidad venezolana con las exigencias del milenio?}

A nivel mundial es conocido que ha habido un crecimiento sustancial y significativo de personas interesadas en cursar estudios a distancia, fenómeno que ha ocurrido también en Venezuela. En consecuencia, las universidades venezolanas se han abocado a cubrir esta necesidad incorporando programas a distancia desde mediados del siglo Xx. Con la propuesta de la Normativa para la Educación Superior a Distancia Nacional, la cual constituiría el marco regulatorio dirigido a garantizar la calidad en la Educación Superior a Distancia. (OPSU, 2007; Dorrego, 2008) se impulsó la modalidad de la educación a distancia; dicha propuesta de normativa está aún sin aprobar. Sin embargo, las IES crearon reglamentos internos que rigen sus sistemas de educación a distancia dentro de un marco legal institucional aprobado, que garantiza la instrumentación de la modalidad, (Mogollón y Vargas, 2012).

Las universidades venezolanas están en una situación particularmente problemática con la disminución de presupuesto para su funcionamiento, desarrollo de investigaciones, migración masiva de personal académico y estudiantil. Es importante señalar que dicha situación obedece a la realidad política, económica y social del país.

En el caso de los sistemas de educación a distancia de las universidades autónomas y universidades privadas, aparece un balance negativo ya que sus cifras de matriculación disminuyen; como, por ejemplo, la Universidad Central de Venezuela (UCv) que muestra un registro histórico en el campus virtual de 16,793 estudiantes, 1,460 docentes y 1,455 cursos. En la actualidad reporta 8,252 estudiantes, 777 docentes y 1,406 cursos. A pesar de lo que se observa en estas cifras, la UCV mediante el Sistema Educación a Distancia de la Universidad Central de Venezuela (SEDUCV) se ha propuesto mantener y proyectar su desarrollo que implica actualización del soporte tecnológico, servicios y talento humano. Para lograr esto es necesario tener el apoyo de empresas privadas, así como establecer alianzas con 
organismos nacionales e internacionales. Esta situación ejemplifica como una de las más importantes universidades del país, continúa trabajando por mantenerse en el actual desarrollo de la educación a distancia.

Ahora bien, por otro lado se observa un crecimiento de ofertas académicas que proceden del campo de la educación continua, se multiplican los entornos virtuales que facilitan cursar este tipo de estudios en línea. En este sentido, como es conocido, el acceso a los estudios universitarios no depende en la actualidad de variables geográficas $\mathrm{y}$, por otro, la enorme cantidad de titulaciones de todo tipo hace que el principal riesgo esté más del lado de la oferta - la conocida como "burbuja" universitaria- que de la demanda. Todo esto aunado a la creciente digitalización e internacionalización del sistema que facilita el acceso de la población a estudios superiores de muy diverso tipo y con planes de financiación flexibles para adaptarse a las necesidades de cada estudiante. Los cursos abiertos masivos online (MOоc) son el ejemplo más destacado de la amplia oferta internacional (Domínguez y Álvarez, 2018).

Los mismos autores comentan que desde octubre de 2011, cuando se ofrecieron los primeros cursos en línea, gratuitos y abiertos al público, más de 900 universidades de todo el mundo habían puesto en marcha cursos gratuitos en línea. A finales de 2018, más de 100 millones de estudiantes se habían inscrito en al menos un curso abierto. Venezuela no escapa de esta realidad, más bien se mantiene en esta corriente internacional de formación.

Las expectativas de avance de la educación a distancia en Venezuela continúan a pesar de las circunstancias de conectividad, infraestructuras tecnológicas que requieren actualización y de otros insumos importantes como la disminución del presupuesto, migración del talento humano y otros aspectos anteriormente mencionados.

\section{Situación venezolana con respecto a Iberoamérica en transformación tecnológica}

La universidades deben dar respuesta a los problemas del mundo, la región y el entorno, por lo que se requiere su incorporación al Sistema de Ciencia e Innovación. En este sentido, existe un informe denominado "La transferencia de I+D, la innovación y el emprendimien- 
to en las universidades. Educación Superior en Iberoamérica" (Barro, 2015). Este tuvo como propósito analizar la transferencia de conocimiento y desarrollo tecnológico, la innovación y el emprendimiento empresarial en las IES iberoamericanas durante la primera década del siglo XXI.

En el mismo se analizan los procesos de valoración de I+D que realizan las universidades de Argentina Brasil, Chile, Colombia, España, México y Portugal. Por otro lado, se dividen los países en grupos a saber: el Grupo 1, constituido por Costa Rica, Cuba, Ecuador, Panamá, Perú y Uruguay y, el Grupo 2, integrado por Bolivia, Guatemala, Honduras, Nicaragua, Paraguay, República Dominicana, El Salvador y Venezuela.

Durante la década 2000-2010, la dotación de los recursos financieros y humanos destinados a I+D por las IEs iberoamericanas ha experimentado un crecimiento significativo. Con respecto a los recursos financieros, todas las IES, con la excepción de Guatemala, vieron aumentado notablemente el gasto total en I+D ejecutado; gasto que se duplicó en la mayoría de países, e incluso se triplicó en los casos de Portugal y Costa Rica, y cuadruplicó en Colombia y Uruguay (Gráfico 1).

Gráfico 1: Gasto total en I+D en las IES en algunos países de la región (2000-2010) Superior 2010 a 200 millones de dólares
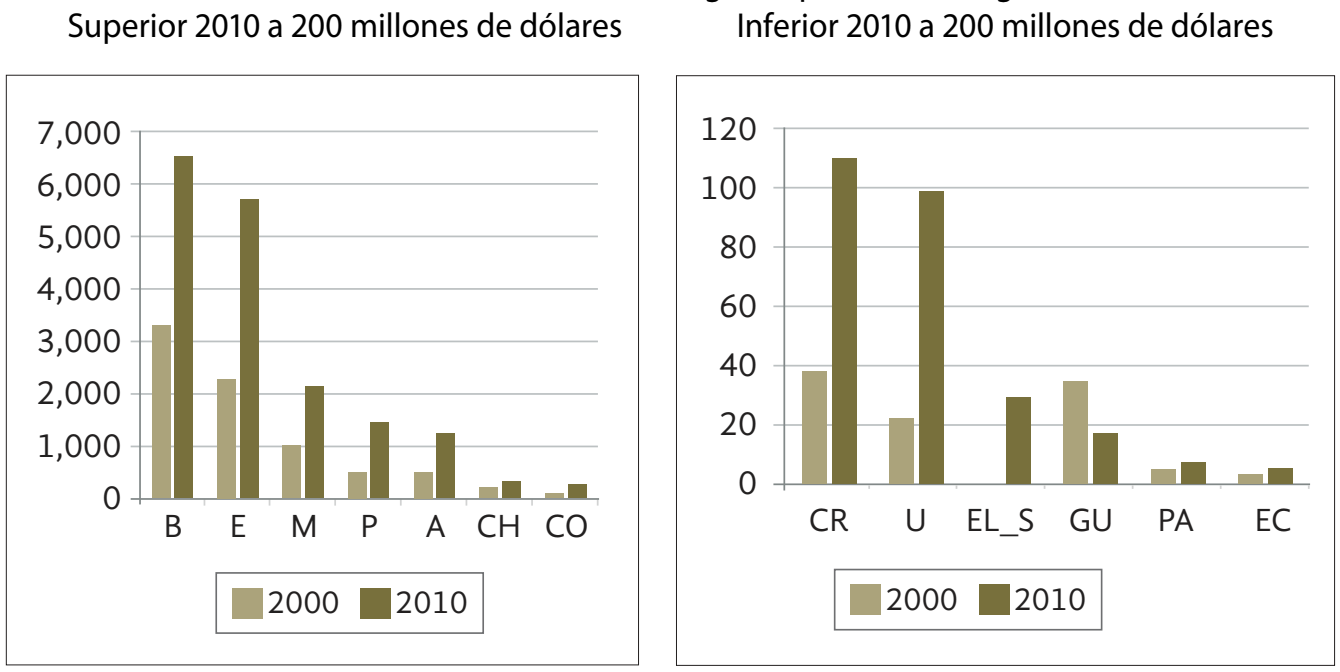

Se observan incrementos en el número de investigadores, que prácticamente se duplicaron en Argentina, Brasil, Colombia y Costa Rica y se triplicaron en Portugal y Venezuela por las políticas de reconocimiento a investigadores, con incrementos más moderados en el resto de países. Estos incrementos, acompañados de una mejora en la calidad de los recursos humanos mediante la incorporación de doctores, obedecieron a diferentes razones: 1) el aumento en las becas doctorales en el caso de Argentina; 2) el crecimiento del número de iEs en los casos de Brasil y México; 3) el diseño de carreras académicas que otorgan cierta estabilidad a los investigadores en el caso de Brasil y de las recientes políticas mexicanas, y 4) políticas de repatriación para atraer a personal investigador que trabajaba en el extranjero en el caso de México (Gráfico 2). 
Gráfico 2. Número total de investigadores en las IES en algunos países de la región (2000-2010)

Superior 2010 a los 5,000 investigadores Inferior 2010 a los 1,500 investigadores
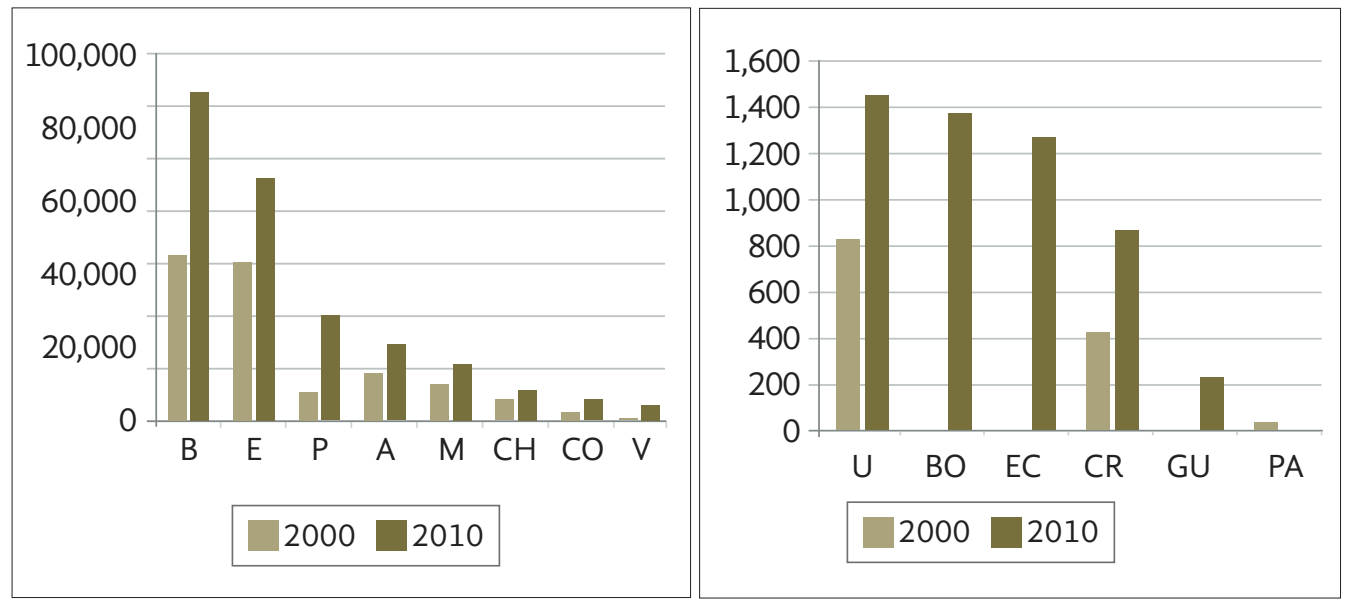

Se destaca la aparición de otro tipo de infraestructura como las incubadoras, los parques científico/tecnológicos y los centros de emprendimiento, cuyo surgimiento dentro de las IES se sitúa en la primera década del siglo XXI.

En términos generales, la segunda infraestructura de apoyo a la transferencia más extendida entre las universidades del estudio es la incubadora. Por su parte, los parques científico/tecnológicos son un centro poco extendido entre las IES, con la excepción de España, donde están presentes en el $40 \%$ de las universidades. Esta desigual distribución en la disponibilidad de parques científico/tecnológicos refleja las distintas políticas de innovación seguidas por los países a nivel nacional, más que las estrategias de las propias IES.

Por otro lado, los centros para facilitar la transferencia de tecnología y resultados de investigación entre la academia $\mathrm{y}$ la industria han adoptado diversas formas jurídicas y organizativas en los distintos países. Sin duda, las que están más presentes, aunque con diversas denominaciones, son las Oficinas de Transferencia de Resultados de Investigación (OTRI) en la península ibérica.

La aparición de estos centros se ha producido de forma progresiva en las IES analizadas, como se observa en la siguiente tabla.

26 Universidades núm. 83, enero-marzo 2020 UDUAL | DOI:https://doi.org/10.36888/udual.universidades.2020.83.73 Ivory Mogollón de Lugo Tendencias, desafíos y desarrollos de la educación a distancia y virtual en la universidad venezolana 
Tabla: Clasificación de las IES en función del porcentaje de universidades que cuentan con OTRI, incubadoras y parques científico/tecnológicos

\begin{tabular}{|c|c|c|c|}
\hline $\begin{array}{c}\% \text { de } \\
\text { Universidades }\end{array}$ & OTRI & Incubadoras & $\begin{array}{l}\text { Parques científico/ } \\
\text { tecnológicos }\end{array}$ \\
\hline$>75 \%$ & $\begin{array}{l}\text { España, México y } \\
\text { Portugal }\end{array}$ & & \\
\hline $51 \%-75 \%$ & & México & \\
\hline $25 \%-50 \%$ & $\begin{array}{l}\text { Brasil, Chile, Colombia y } \\
\text { Uruguay }\end{array}$ & $\begin{array}{l}\text { Brasil, Chile, Colombia, } \\
\text { España y Uruguay }\end{array}$ & España \\
\hline$<25 \%$ & $\begin{array}{l}\text { Grupo 1: Costa Rica, } \\
\text { Cuba, } \\
\text { Ecuador, Panamá y Perú } \\
\text { Grupo 2: Bolivia, } \\
\text { Guatemala, } \\
\text { Honduras, Nicaragua, } \\
\text { Paraguay, República } \\
\text { Dominicana, El Salvador y } \\
\text { Venezuela }\end{array}$ & $\begin{array}{l}\text { Grupo 1: Costa Rica, } \\
\text { Cuba, } \\
\text { Ecuador, Panamá y Perú } \\
\text { Grupo 2: Bolivia, } \\
\text { Guatemala, } \\
\text { Nicaragua, Paraguay, } \\
\text { República Dominicana y } \\
\text { Venezuela }\end{array}$ & $\begin{array}{l}\text { Brasil, Colombia y México } \\
\text { Grupo 1: Cuba, Ecuador, } \\
\text { Panamá y Uruguay } \\
\text { Grupo 2: Bolivia, } \\
\text { Honduras, } \\
\text { Nicaragua, Paraguay, } \\
\text { República Dominicana, El } \\
\text { Salvador y Venezuela }\end{array}$ \\
\hline No consta & & $\begin{array}{l}\text { Grupo 2: Honduras y El } \\
\text { Salvador }\end{array}$ & $\begin{array}{l}\text { Grupo 1: Costa Rica y } \\
\text { Perú } \\
\text { Grupo 2: Guatemala }\end{array}$ \\
\hline
\end{tabular}

Es importante señalar que el informe analiza la transferencia de conocimiento y desarrollo tecnológico y la innovación en las IES iberoamericanas durante la primera década del siglo XXI de los países Argentina, Brasil, Chile, Colombia, España, México, Portugal, Bolivia, Guatemala, Honduras, Nicaragua, Paraguay, República Dominicana, El Salvador y Venezuela.

Como se puede apreciar, Venezuela ha tenido presencia y participación en los estándares mundiales relacionados con los procesos de valorización de I+D.

\section{Apuesta para el presente y futuro de la universidad venezolana}

Así como en la actualidad gobiernos nacionales alrededor del mundo han lanzado sus propias plataformas de cursos abiertos, en Venezuela también existen procesos de cambio, de gestión, de estructura que muestran los modelos y sistemas de educación a distancia, producidos en los últimos tiempos en el área cultural, económica y política. Así como también el rápido desarrollo de los conocimientos, el progreso de los medios tecnológicos al servicio de la información, el acceso de la población a una mayor disposición de tiempo y de bienes.

La demanda de actualización científica, tecnológica y humanística que se está experimentando en la sociedad ha permitido identificar en Venezuela que las IES requieren modelos de enseñanza flexibles, capaces de renovar y transmitir conocimientos y técnicas que proporcionen respues- 
ta a esa demanda educativa. Las universidades venezolanas están en un proceso avanzado, reportan la incorporación de modelos institucionales a distancia. La UCV es parte de este desarrollo inminente que lleva a las instituciones venezolanas hacia la bimodalidad con un avance significativo en estos últimos cinco años (Hernández y Mogollón, 2018) como la incorporación de programas de postgrados al campus virtual, el fortalecimiento de desarrollo de aplicaciones web que facilitan los procesos académicos y administrativos del sistema.

La Universidad Nacional Abierta reporta, en este sentido, que como espacio socioeducativo provee de condiciones fundamentales para el despliegue de cambios sustanciales en el estilo de vida de los estudiantes. Cabe destacar que esta institución universitaria cumple con los principios sobre los cuales se erige, especialmente en lo atinente a la innovación como posibilidad de ofrecer una educación acorde con las necesidades e intereses de los estudiantes y con el principio del desarrollo autónomo para incrementar la calidad de vida del ciudadano venezolano (Leal, 2018).

La Universidad de Carabobo menciona que, si bien se conoce que ha avanzado en cuanto a la implementación de la educación superior a distancia como nuevo paradigma del poder de la información, reconoce que progresivamente se incrementarán contenidos virtuales y programas a distancia. La intención es continuar con la capacitación de los docentes en las competencias técnico-didácticas para impartir la educación a distancia, con el fin de innovar y aportar a los principios de la calidad educativa en esta modalidad (Romero, Fiori y Páez, 2018).

La Universidad de los Llanos Occidentales Ezequiel Zamora ofrece clara evidencia de los cambios que ha experimentado la educación a distancia con el uso de aulas virtuales como apoyo al modelo presencial, la facilitación de diferentes cursos bajo modalidades mixtas o híbridas, generación de trabajos de investigación en línea, la mediación con tecnologías de la información y la comunicación y la necesidad de incorporar infraestructuras adaptadas a las nuevas tecnologías. Se espera que el programa de formación se constituya como una política institucional para ampliar su cobertura hacia otros contextos universitarios (Azuaje, León y Amario, 2018).

Por su lado, la Universidad Pedagógica Experimental Libertador (UPEL) señala que en los últimos años se ha intentado avanzar en este sentido, procurando hacer énfasis tanto en los aspectos tecnológicos como en los pedagógicos, aún dentro de las limitaciones económicas que el país enfrenta y los recortes presupuestarios en las universidades. Uno de sus avances es el Programa de Tecnología de Información y Comunicación o PROTIC, a cargo del Vicerrectorado de Extensión de la UPEL, y que fue fundado con el propósito de promover actividades tendientes a la aplicación de las TIC al ámbito intra y extra universitario. Entre los objetivos centrales de dicho programa destacan: a) la realización de actividades formativas, actualización y desarrollo profesional enmarcadas en ambientes virtuales y mediados por las tecnologías digitales; b) estimulación del uso de materiales educativos impresos y digitales que se adapten a los contextos de capacitación académica bajo la educación a distancia y, c) orientación y promoción de proyectos de radio y televisión universitarios con fines educativos. Otro avance importante fue la creación de su plataforma El Salón Virtual de la 
UPEL y el desarrollo del marco legal de referencia para la implementación de la educación a distancia, entre otros (Suárez, 2018).

Como se observa, la educación superior a distancia en Venezuela continua con un desarrollo diverso y plural ha tenido avances relacionados con aspectos epistemológicos y teóricos de la educación a distancia, estructuras organizacionales, marcos legales, formación de docentes y desarrollos de campus virtuales.

Uno de los señalamientos que indica el futuro de la educación lo encontramos en la Declaración de Incheon, Educación 2030 (2015), la cual ofrece una amplia visión que apunta a transformar las vidas mediante la educación, reconociendo el importante papel que desempeña la educación como motor principal del desarrollo y para la consecución de los Objetivos de Desarrollo del Milenio. Se realiza un compromiso con carácter de urgencia con una agenda de la educación única y renovada que sea integral, ambiciosa y exigente, sin dejar a nadie atrás. Con la intención de garantizar una educación inclusiva, equitativa y de calidad, y promover oportunidades de aprendizaje durante toda la vida para todos.

Otro aspecto que se señala es el compromiso a promover oportunidades de aprendizaje de calidad en todos los contextos y en todos los niveles educativos. Ello incluye un mayor acceso en condiciones de igualdad a la enseñanza y formación técnica y profesional de calidad, a la educación superior y a la investigación, prestando la debida atención a la garantía de la calidad, además de ofrecer vías de aprendizaje flexibles y fortalecer la ciencia, la tecnología y la innovación. Es preciso aprovechar las tecnologías de la información y la comunicación para reforzar los sistemas educativos, la difusión de conocimientos, el acceso a la información, el aprendizaje efectivo y de calidad, y una prestación más eficaz de servicios.

También se declara que es preciso desarrollar sistemas de educación más inclusivos, que ofrezcan mejores respuestas y que tengan una mayor capacidad de adaptación para satisfacer las necesidades que presenta la sociedad del milenio. Se subraya la necesidad de que la educación se imparta en entornos de aprendizajes sanos y seguros.

En conclusión, se mencionan informes y declaraciones importantes que señalan cuál es el futuro de las IES a nivel mundial. Las universidades venezolanas deben responder a las necesidades de la sociedad del milenio para estar acorde con el desarrollo de la educación superior a distancia mundial en la medida de sus posibilidades y en concordancia con lo manifestado en el Informe Horizon, los lineamientos de la UNESCO y la Educación 2030.

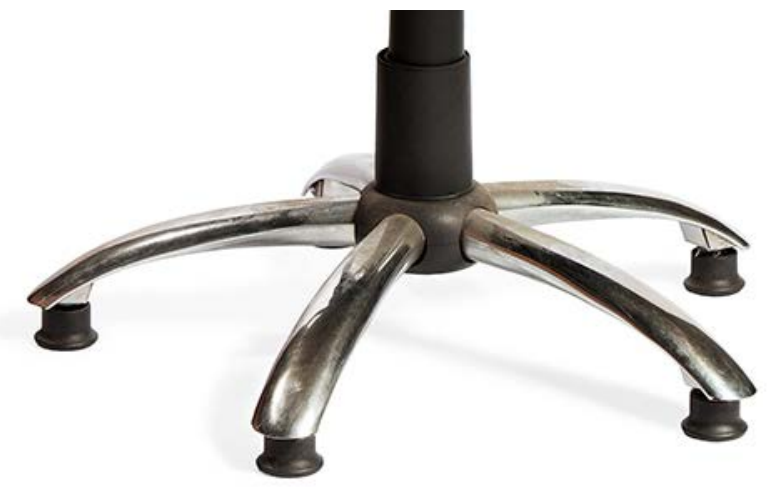




\section{Referencias}

Azuaje, D.; León D. y Amario L. (2018). Programa de formación en entornos virtuales de enseñanza y aprendizaje (EVEA) de la UNELLEZ. En Asociación Venezolana de Educación a Distancia. Educación a Distancia en Venezuela. Disponible en: http://aved.net.ve/OBRA_AVED.pdf

Barro, S. (Coord.) (2015). La transferencia de I+D, la innovación y el emprendimiento en las universidades. Educación superior en Iberoamérica. Informe 2015. Chile: CINDA.

Cruz, A. (2014). Análisis de las Actividades de Investigación + Desarrollo + Innovación + Emprendimiento en Universidades de Iberoamérica, Colección Estudios RedEmprendia.

Foro Mundial sobre la Educación. (2015). Declaración de Incheon, Educación 2030: Hacia una educación inclusiva y equitativa de calidad y un aprendizaje a lo largo de la vida para todos. Disponible en: https://unesdoc.unesco.org/ark:/48223/pf0000233137_spa

Domínguez, D., y Álvarez, J.F. (2019). Structural changes in the landscape of Spanish distance universities. Open Praxis, 11(2), 119-128. Disponible en: https://doi.org/10.5944/openpraxis.11.2.958

Dorrego, E. (2008). La Educación a distancia en Venezuela. Realidades y tendencias en Mena, M.; Rama; C y Facundo, A. El Marco regulatorio de la Educación Superior a Distancia. UNAD, VIRTUAL EDUCA, ICDE.

Hernández, Y. y Mogollón I. (2018). Modelo institucional de la educación superior a distancia mediada por las TIC en Venezuela: hacia la Universidad Bimodal. En Asociación Venezolana de Educación a Distancia. Educación a Distancia en Venezuela. Disponible en: http://aved.net. ve/OBRA_AVED.pdf

Informe Horizon (2019). Disponible en: https://bit.ly/2HYAIDY

Leal, N. (2018). Estudios universitarios a distancia y cambios en el estilo de vida. En Asociación Venezolana de Educación a Distancia. Educación a Distancia en Venezuela. Disponible en: http:// aved.net.ve/OBRA_AVED.pdf

Mogollón, I y Vargas, M.L. (2012). Proyecto Nacional de Educación a Distancia de Venezuela. Visión Dra. Elena Dorrego. En Mogollón, I. (2012) Venezuela, Educación a Distancia, Encuentros protagonistas y experiencias. Disponible en: https:/www.edutec.es/publicaciones/ educacion-distancia-encuentros-protagonistas-experiencias

Mogollón, I. (2016). La educación a distancia en Venezuela. Una realidad. En Cruz, M y Rama, C. La educación a distancia y virtual en Centroamérica y el Caribe. República Dominicana. Editorial Buho. SRL

Mogollón, I y Rama, C. (2017). Impacto de las tecnologías en la educación superior a distancia en Venezuela. Una época de retos. Sistema de Educación a Distancia de la Universidad Central de Venezuela. VIRTUAL EDUCA. Disponible en: https://virtualeduca.org/observatorio

OPSU, (2007). Proyecto Nacional de Educación Superior a Distancia. Caracas, Venezuela.

Romero, Y.; Fiori, M. y Páez M. (2018). Educación a distancia, nuevo paradigma del poder de la información en sector universitario. En Asociación Venezolana de Educación a Distancia. Educación a Distancia en Venezuela. Disponible en: http://aved.net.ve/OBRA_AVED.pdf

Suárez, Y. (2018). Educación a distancia en la UPEL. camino recorrido y prospectiva. En Asociación Venezolana de Educación a Distancia. Educación a Distancia en Venezuela. Disponible en: http://aved.net.ve/OBRA_AVED.pdf 